\title{
A PROOF of Morse's THEOREM ABOUT THE CANCELlATION OF CRITICAL POINTS
}

\author{
François Laudenbach
}

\begin{abstract}
In this note, we give a proof of the famous theorem of M. Morse dealing with the cancellation of a pair of non-degenerate critical points of a smooth function. Our proof consists of a reduction to the one-dimensional case where the question becomes easy to answer.
\end{abstract}

\section{INTRODUCTION}

Let us consider an $n$-dimensional closed manifold $M$ equipped with a Morse function $f$ : $M \rightarrow \mathbb{R}$. Marston Morse devoted two papers to the question of canceling a pair of critical points. In [6] he considered the possibility of canceling a local minimum (index 0) with a critical point of index 1 ; and, similarly the cancellation of a pair of indices $(n-1, n)$; in that paper, a polar function means a function with no supernumerary local extrema. In [7] (see also [2) Morse extended its cancellation criterion to the case of a pair of indices $(k, k+1)$. It is worth noticing that these two papers show the first time where Morse is considering gradients globally in the manifold $M$, in the spirit of the 1949 Note by René Thom [11].

S. Smale generalized Morse's criterion and got a more algebraic criterion for cancellation. This generalization became an important tool in Smale's proof of the generalized Poincaré conjecture [10]. A detailed proof is offered by J. Milnor in [5] where the cancellation theorem of Morse occupies one chapter (i.e. Section 5) and is told to be "quite formidable".

Actually, Milnor's proof deals with the cancellation of two zeroes of a gradient (or pseudogradient) allowing the deformation to run among vector fields which a priori are not gradients. The effective deformation of the function remains hidden and the crossing of the codimension one stratum of functions with one cubic singularity is invisible. Our goal is to make this deformation visible; in particular the support of the deformation will be specified, as it is in the notes by J. Cerf \& A. Gramain [1] (actually, we specify a smaller support than in [1] where some saturation is not useful). Moreover, we intend to show that the question of cancellation reduces to the one-dimensional case where it is easy to solve. The same technique was already used in [3].

The statement we give here is slightly different from the one in [5] which will be derived as a corollary. Moreover, we work with descending pseudo-gradient, called pseudo-gradient for short. According to K. Meyer [4], it is a vector field $X$ satisfying the two following conditions:

- Lyapunov inequality: $X \cdot f<0$ apart from the critical points of $f$;

- Non-degeneracy condition: $X \cdot f$ has a non-degenerate maximum at each critical point of $f$.

Such a pseudo-gradient $X$ may be built by partition of unity. It follows from the definition that if $p$ is a critical point of $f$, it is a hyperbolic zero of $X$ and hence, there are stable and unstable manifolds, respectively denoted by $W^{s}(p)$ and $W^{u}(p)$, which are formed by the points $x \in M$ such that $X^{t}(x)$ tends to $p$ as $t$ tends to $+\infty$ or $-\infty$; here $X^{t}$ denotes the flow of $X$. The 
dimension of the unstable manifold $W^{u}(p)$ and the codimension of the stable manifold $W^{s}(p)$ both equal the index of the critical point $p$. It is easily checked that the restriction of $f$ to $W^{u}(p)$ (resp. $W^{s}(p)$ ) has a non-degenerate maximum (resp. minimum).

TheOREM. Let us consider the Morse function $f: M^{n} \rightarrow \mathbb{R}$ equipped with a pseudo-gradient $X$. Let $p$ and $q$ be two critical points of $f$ satisfying the following conditions:

i) $W^{u}(p)$ and $W^{s}(q)$ intersect transversely and the intersection is made of one orbit $\ell$ of $X$ only.

ii) For some $\varepsilon>0$, each orbit of $X$ in $W^{u}(p)$ distinct from $\ell$ crosses the level set $f^{-1}(f(q)-\varepsilon)$.

Then the pair $(p, q)$ is cancelable. More precisely, if $U$ denotes an open neighborhood of the closure of $W^{u}(p) \cap\{f \geq f(q)-\varepsilon\}$, there is a path of smooth functions $\left(f_{t}\right)_{t \in[0,1]}$, such that:

1) $f_{0}=f$;

2) for every $t \in[0,1], f_{t}$ coincides with $f$ on $M \backslash U$;

3) the function $f_{t} \mid U$ is Morse with two critical points when $0 \leq t<1 / 2$; it has a cubic singularity when $t=1 / 2$ and it has no critical point when $1 / 2<t \leq 1$.

By computing the dimensions one has the following formula for the Morse indices: ind $(p)=$ $\operatorname{ind}(q)+1$. There are other statements by varying assumption ii) which can be derived from the above theorem.

\section{Proof of the theorem}

Say $\operatorname{index}(p)=k+1=\operatorname{index}(q)+1$. We are given an open neighborhood $U$ of the closure of $W^{u}(p) \cap\{f(q) \leq f \leq f(p)\}$. After looking at $U \cap\{f=f(q)\}$, we may assume that the frontier of $U$ in $M$ traces a cylinder $C$ tangent to $X$ in the domain $\left\{f(q)-\varepsilon^{\prime} \leq f \leq f(q)+\varepsilon^{\prime}\right\}$, where $\varepsilon^{\prime}>0$ satisfies assumption ii) of our theorem. We then choose Morse charts $U(p)$ and $U(q)$ about $p$ and $q$ respectively, both contained in $U$. They are equipped with coordinates $(\tilde{y}, z) \in \mathbb{R}^{k+1} \times \mathbb{R}^{n-k-1}$ (resp. $\left.(y, \tilde{z}) \in \mathbb{R}^{k} \times \mathbb{R}^{n-k}\right)$ such that $\left.f|U(p)=f(p)-| \tilde{y}\right|^{2}+|z|^{2}$ (resp. $\left.\left.f|U(q)=f(q)-| y\right|^{2}+|\tilde{z}|^{2}\right)$. Moreover, it is possible to require that $(\tilde{y}, 0)$ are coordinates of $W^{u}(p) \cap U(p)$ (thus, $z$ must be coordinates in the orthogonal complement to $T_{p} W^{u}(p)$ with respect to the Hessian of $f$ ). Similarly, the coordinates of $U(q)$ may be chosen so that $(0, \tilde{z})$ are coordinates of $W^{s}(q) \cap U(q)$.

For $0<\varepsilon<\varepsilon^{\prime}$ small enough there is a Morse model $\mathbb{M}(q) \subset U(q)$ disjoint from $C$ whose top and bottom are respectively at level $f(q)+\varepsilon$ and $f(q)-\varepsilon$; and also, there is a Morse model $\mathbb{M}(p) \subset U(p)$ with top and bottom at level $f(p)+\varepsilon$ and $f(p)-\varepsilon$.

Denote by $\tilde{\ell}$ the trace of $\ell$ in the domain $\{f(q)+\varepsilon \leq f \leq f(p)-\varepsilon\}$. One end point $a$ of $\tilde{\ell}$ is $W^{u}(p) \cap\{f=f(p)-\varepsilon\} \cap W^{s}(q)$ and the other end point $b$ is $W^{u}(p) \cap\{f=f(q)+\varepsilon\} \cap W^{s}(q)$. Consider the diameter $\Delta_{a} \subset U(p)$ of the ball $\left\{|\tilde{y}|^{2} \leq \varepsilon, z=0\right\}$ ending at $a$. Split the coordinates $(\tilde{y}, z)$ as $(t, y, z)$ so that $t$ is a coordinate of $\Delta_{a}$ and

$$
\left.f\left|U(p)=f(p)-t^{2}-\right| y\right|^{2}+|z|^{2} .
$$

Similarly, split $(y, \tilde{z})=(y, t, z)$ in $U(q)$ so that $b \in \Delta_{b}:=\{y=0, z=0\}$ and

$$
\left.f|U(q)=f(q)-| y\right|^{2}+t^{2}+|z|^{2} .
$$

It is easy to find a $C^{\infty}$ parametrized arc $\alpha:[0,1] \rightarrow M, u \in[0,1] \mapsto \alpha(u) \in M$, whose image is denoted by $A$, and a sequence $0<u_{0}<u_{0}^{\prime}<u_{1}^{\prime}<u_{1}<1$ with the following properties: 
- $p=\alpha\left(u_{0}\right), a=\alpha\left(u_{0}^{\prime}\right), b=\alpha\left(u_{1}^{\prime}\right), q=\alpha\left(u_{1}\right)$ and $\alpha\left(\left[u_{0}^{\prime}, u_{1}^{\prime}\right]\right)=\tilde{\ell}$;

- the image $\alpha\left(\left[0, u_{1}^{\prime}\right]\right)$ is contained in $W^{u}(p)$ and the image $\alpha\left(\left[u_{1}^{\prime}, 1\right]\right)$ is contained in $W^{s}(q)$;

- the function $h$ defined by $h:=f \circ \alpha$ has two critical points, one maximum in $p$, one local minimum in $q$ (both non-degenerate) and varies from $h(0)=f(q)-\varepsilon$ to $h(1)=f(q)+\varepsilon$.

One observes that there exists an increasing smooth function $h_{1}$ without critical points, which coincides with $h$ near the extremities of $[0,1]$, and satisfies

$$
\text { (*) } \quad h_{1} \leq h .
$$

This is the solution of the cancellation problem in the one-dimensional case.

We now construct a $(k+1)$-dimensional sub-manifold $W \subset U$ containing $A$ such that $p$ and $q$ are the only critical points of $f \mid W$. This $W$ will contain the part of $W^{u}(p)$ above the level $f=f(q)+\varepsilon$ that is, $W^{u}(p) \cap\{f \geq f(q)+\varepsilon\} \cong D^{k+1}$. Let $S$ be the boundary of this disc. Let $T$ denote the top of $\mathbb{M}(q)$; we have $T \cong D^{k} \times S^{n-k-1}$ and the product structure of $T$ is determined by the Morse coordinates. The point $b$ belongs to the so-called belt sphere $\{0\} \times S^{n-k-1} \subset W^{s}(q)$. The radius of $D^{k}$ measured with the norm of the $y$-coordinates is a free choice for the Morse model $\mathbb{M}(q)$. If this radius is small enough, the transversality assumption implies that $S \cap T$ is isotopic in $T$ to the standard meridian $D^{k} \times\left\{b_{0}\right\}$ by an isotopy keeping the belt sphere fixed. Therefore, by a first level preserving isotopy of the coordinates of $\mathbb{M}(q)$ one makes $S \cap T$ be contained in $\{z=0\}$. By a second isotopy of this type one makes $X$ and $\nabla f$ coincide near the top of $\mathbb{M}(q)$; here $\nabla f$ stands for the descending gradient of $f$ in $\mathbb{M}(q)$ associated to the Euclidean metric of the coordinates $(t, y, z)$. These two isotopies leave $W^{s}(q)$ invariant and are stationary near $q$.

Let $B \subset \mathbb{M}(q)$ be defined by $B:=\{z=0\}$; it is $C^{\infty}$ tangent to $W^{u}(p)$ along $B \cap S$. By partition of unity one finds a pseudo-gradient $\xi$ such that:

- $\xi=X$ on $W^{u}(p)$ above the level $f=f(q)+\varepsilon$ and on the cylinder $C$ defined previously;

- $\xi=\nabla f$ in $\mathbb{M}(q)$.

Let $W_{\xi}^{u}(p)$ be the unstable manifold of $p$ for $\xi$. Each orbit of $\xi$ in $W_{\xi}^{u}(p)$ which does not meet $\tilde{\ell}$ reaches the level set $\{f=f(q)-\varepsilon\}$ and remains in $U$ since $C$ prevents it from getting out. We define $W$ as the union of $B$ and $W_{\xi}^{u}(p)$ truncated by removing $\{f<f(q)-\varepsilon\}$.

LEMmA 1. There exist a tubular neighborhood $N$ of $A$ in $U$ and coordinates $(u, y, z) \in[0,1] \times$ $\mathbb{R}^{k} \times \mathbb{R}^{n-k-1}$ of $N$, such that:

i) $A=\{y=0, z=0\}$;

ii) $N_{0}:=N \cap W=\{z=0\}$;

iii) $f(u, y, z)=h(u)-|y|^{2}+|z|^{2}$ at every point of $N$.

ProOf. The first two items are easy to realize: take a provisory pair of small tubular neighborhoods $\left(N, N_{0}\right)$ of $A$ in $(M, W)$ whose fibres are contained in $\{t=$ const $\}$ with respect to the coordinates of $\mathbb{M}(p)$ and $\mathbb{M}(q)$. Choose the coordinates $y$ in the fibre of $N_{0}$ over $A$ and $z$ in the fibre of $N$ over $N_{0}$ such that $(y, z)$ are the so-named coordinates in $\mathbb{M}(p)$ and $\mathbb{M}(q)$. For proving item iii), we need to invoke a general fact stated below. 
Claim. Let $V$ be a smooth manifold and $V^{\prime} \subset V$ be a sub-manifold. Two germs of smooth functions $f$ and $g$ along $V^{\prime}$ whose restrictions to $V^{\prime}$ coincide and have no critical points are isotopic relative to $V^{\prime}$ (meaning that they become equal after moving one of them by an isotopy of $V$ fixing $\left.V^{\prime}\right)$; moreover, if $f=g$ near a compact set $K \subset V^{\prime}$, the isotopy may be the identity near $K$.

For proving this claim the path method of Moser is available; it may be applied to the path $t \in[0,1] \mapsto f_{t}:=(1-t) f+t g$, that is: there is a time-dependent vector field $Z_{t}$ whose integration solves the problem of conjugating each $f_{t}$ to $f_{0}$ near $V^{\prime}$ smoothly in $t \in[0,1]$. It is sufficient to find $Z_{t}$ satisfying $d f_{t}(x) \cdot Z_{t}(x)=f(x)-g(x)$ near $V^{\prime}$ (see [8]); for that, apply the implicit function theorem for finding local solutions which are then glued by partition of unity.

We are going to apply this claim twice. First, apply it to the data: $V=W, V^{\prime}=A$, $K=A \cap(\mathbb{M}(p) \cup \mathbb{M}(q))$, the restriction $f \mid N_{0}$ and $g(u, y)=h(u)-|y|^{2}$. The isotopy yielded by the claim moves the coordinates $(u, y)$, without changing $u$ on $A$, so that the equality of iii) holds true in a neighborhood $N_{1} \subset N_{0}$ of $A$ in $W$. Then, apply the claim to the data: $V=M$, $V^{\prime}=N_{1}, K=N_{1} \cap(\mathbb{M}(p) \cup \mathbb{M}(q)), f$ and $g(u, y, z)=h(u)-|y|^{2}+|z|^{2}$. The new isotopy finishes to put the coordinates in a position which makes the equality of item iii) hold true near $A$ in $M$ yielding the desired $N$.

In $W \cap N$, the vector field $Y:=y \partial_{y}$ is tangent to $W$ and it is a pseudo-gradient for $f \mid(W \cap$ $(N \backslash A))$. Still denoted $Y$, it extends to the whole $W$ as a pseudo-gradient for $f \mid(W \backslash A)$ (by partition of unity). Each orbit of $Y$ converge to a point of $A$ in the past. This defines a $k$-disk fibration $W \rightarrow A$ which is pinched at $\alpha(0) \in A$. Indeed, this fibration is clear for $W \cap N \rightarrow A$ and the orbits of $Y$ yield an ambient isotopy from $W$ to $W \cap N$. Denote $D_{u}$ the fibre of $W$ over $\alpha(u)$.

Let $\widetilde{W}$ be a tubular neighborhood of $W$ in $\{f \geq f(q)-\varepsilon\}$. The fibration $W \rightarrow A$ extends to $\widetilde{W}$ as a $(n-1)$-disk fibration:

$$
\widetilde{D}_{u} \hookrightarrow \widetilde{W} \rightarrow A
$$

pinched at $\alpha(0)$ and coinciding with the projection $(u, y, z) \rightarrow \alpha(u)$ in $N$. The function $f_{u}:=f \mid \widetilde{D}_{u}$ is a Morse function with $\alpha(u)$ as unique critical point and its index is $k$. It has a pseudo-gradient $\widetilde{Y}_{u}$, tangent to $\widetilde{D}_{u}$, which coincide with $Y$ on $W$ and with $y \partial_{y}-z \partial_{z}$ on $N$.

LEMMA 2 (Decreasing of a critical value.) Let $g: V \rightarrow \mathbb{R}$ be a Morse function with a pseudogradient $Z$. Let $p$ be a critical point of index $k$ and let $a<g(p)$. Assume that the unstable manifold $W^{u}(p)$ contains a compact $k$-disk $D$ whose boundary lies in the level set $\{g=a\}$. Let $U$ be a neighborhood of $D$ in $\{g \geq a\}$. Then, for every $\varepsilon>0$, there exists a one parameter family of Morse functions $\left(g_{t}\right)_{t \in[0,1]}$, such that:

- $g_{0}=g, a<g_{1}(p)<a+\varepsilon$,

- $Z$ is a pseudo-gradient of $g_{t}$ for every $t \in[0,1]$,

- and $g_{t}=g$ out of $U$. 
The same statement holds true with parameters.

ProOF. The point $p$ is a hyperbolic zero of the vector field $Z$. Then, the orbits of $Z$ close to $D$ but not tangent to $D$ are crossing the level set $\{g=g(p)+\eta\}$, for a small $\eta>0$ and, of course, the level set $\{g=a\}$. Consider the foliation $\mathcal{F}$ defined by $g=$ const on $U \backslash D$. Find an $n$-dimensional compact domain $K$ in $U \backslash D$ made of pieces of $Z$-orbits from $\{g=g(p)+\eta\}$ to $\{g=a\}$ surrounding $D$. It is easy to replace $\mathcal{F} \mid K$ by a foliation whose leaves are still transverse to $Z$ in order to have the level sets of the wanted function $g_{1}$. We refer to [3] for more details (see also [5] Section 4 or [9] Section 2).

End OF THE PROOF OF THE THEOREM. The critical point of $f_{u}$ is $p_{u}:=\alpha(u)=(u, 0,0)$ and its value is $h(u)$. The vector field $\widetilde{Y}_{u}$ is a pseudo-gradient for $f_{u}$ and the disk $D_{u}$ fulfills the requirement of Lemma 2, which we apply smoothly with respect to the parameter $u$. So, it is possible to decrease the critical value of $f_{u}$, smoothly in $u \in[0,1]$, from $h(u)$ to $h_{1}(u)$, still keeping $\widetilde{Y}_{u}$ as a pseudo-gradient. Therefore, the deformation of $f$ over $A$ from $h$ to $h_{1}$ (compare $(*)$ ), extends to $\widetilde{W}$ without creating new critical points away from $A$. Moreover, the deformation is supported in $\widetilde{W}$, hence in $U$, as wanted.

\section{ApplicAtions}

In this section, there are given two more classical statements, starting with the one from Milnor's book ([5], Section 5).

Corollary 1. Let $\left(W, L_{0}, L_{1}\right)$ be a compact cobordism and $f:\left(W, L_{0}, L_{1}\right) \rightarrow([0,1], 0,1)$ be a Morse function with two critical points $p$ and $q$. Assume there is a pseudo-gradient such that $W^{u}(p)$ and $W^{s}(q)$ intersect in one orbit and transversely. Then the cobordism is a product: $W \cong M_{0} \times[0,1]$.

ProOF. Certainly $0<f(q)<f(p)<1$. By compactness, every orbit of the pseudo-gradient in $W^{u}(p)$ distinct from the connecting orbit reaches $L_{0}$. Hence, the assumptions of our theorem are fulfilled. After canceling the critical points, $W$ is a product by gradient lines.

Corollary 2. Let $M$ be a closed manifold, $f: M \rightarrow \mathbb{R}$ be a Morse function, $(p, q)$ be a pair of critical points whose respective indices are $k+1$ and $k$. Let $X$ be a pseudo-gradient of $f$. Assume that there are compact disks $D(p) \subset W^{u}(p)$ and $D(q) \subset W^{s}(q)$ with the following properties:

- $D(p)$ and $D(q)$ are neighborhoods of $p$ and $q$ respectively in $W^{u}(p)$ and $\left.W^{s}(q)\right)$;

- their boundaries lie in a regular level set $\{f=a\}, f(q)<a<f(p)$ in which they intersect in one point only and transversely.

Then, the pair $(p, q)$ is cancelable. 
Proof. Since $a$ is a regular value, the disk $D(q)$ can be extended (keeping its name) so that its boundary lies in $\{f=a+\varepsilon\}$, for some small $\varepsilon>0$. Lemma 2 can be applied for decreasing the critical value $f(p)$ so that $a<f(p)<a+\varepsilon$. Similarly, by considering $-f$, Lemma 2 can be applied for increasing the critical value $f(q)$ so that $a<f(q)<f(p)<a+\varepsilon$. In this situation, as in Corollary 1, the assumptions of our theorem are fulfilled. The cancellation follows.

\section{REFERENCES}

[1] J. Cerf, A. Gramain, Le théorème du h-cobordisme (Smale), cours Orsay 1966, Secr. math. Éc. Normale Sup., Paris, 1968 (www.maths.ed.ac.uk/ aar/surgery/cerf-gramain.pdf).

[2] W. Huebsch, M. Morse, The bowl theorem and a model non-degenerate function, Proc. Nat. Acad. Sc. U.S.A., vol. 51 (1964), 49-51.

[3] F. Laudenbach, A proof of Reidemeister-Singer's theorem by Cerf's methods, arXiv(math-GT): 1202.1130.

[4] K. Meyer, Energy functions for Morse-Smale systems, Amer. J. Math. 90 (1968), 1031-1040.

[5] J. Milnor, Lectures on the h-cobordism theorem, Princeton Univ. Press, 1965.

[6] M. Morse, The existence of polar non-degenerate functions on differentiable manifolds, Annals of Math. 71 (1960), 352-383.

[7] M. Morse, Bowls of a non-degenerate function on a compact differentiable manifold, 81-103 in: Differential and Combinatorial Topology (A Symposium in Honor of Marston Morse), Princeton Univ. Press, 1965.

[8] J. Moser, On the volume elements on a manifold, Trans. Amer. Math. Soc. 120 (1965), 286-294.

[9] S. Smale, On gradient dynamical systems, Annals of Math. 74 (1961), 199-206.

[10] S. Smale, Generalized Poincaré's conjecture in dimensions greater than four, Annals of math. 74 (2) (1961), 391-406.

[11] R. Thom, Sur une partition en cellules associée à une fonction sur une variété, Comptes Rendus Acad. Sc. Paris, t. 228, (1949), 973-975.

Laboratoire de Mathématiques Jean Leray, UMR 6629 du CNRS, Faculté des Sciences et Techniques, Université de Nantes, 2, rue de la Houssinière, F-44322 Nantes cedex 3, France.

E-mail address: francois.laudenbach@univ-nantes.fr 